\title{
ALARM COMPLIANCE IN HEALTHCARE: DESIGN CONSIDERATIONS FOR ACTIONABLE ALARMS (IN INTENSIVE CARE UNITS)
}

\author{
Sanz-Segura, Rosana (1); Manchado Pérez, Eduardo (1); Özcan, Elif (2) \\ 1: School of Engineering and Architecture. University of Zaragoza; 2: Faculty of Industrial Design \\ Engineering Delft University of Technology. Department of Intensive Care Erasmus Medical Centre
}

\begin{abstract}
Intensive care units are technologically advanced environments that are designed to safeguard the patient while their vitals are stabilized for further treatment. Audible and visual alarms are part of the healthcare ecology. However, these alarms are so many that clinicians suffer from a syndrome called 'alarm fatigue' and often do not comply with the task alarm is conveying. Measuring compliance with rules in the workspace and determining the success of a system belongs to the field of ergonomics and is based on data collected through task observations and scoring. In this paper, we will explore compliance with critical alarms by not only from their potential success or failure perspective but also from the perspectives of the clinician capacity, needs, and motivations to comply with alarms in critical environments. We will finally, reflect on further possible design strategies to increase compliance in critical care that are beyond following rules per se but through intrinsic motivation.
\end{abstract}

Keywords: Compliance, Design practice, Decision making, Audible alarms, Design engineering

Contact:

Sanz-Segura, Rosana

University of Zaragoza

Design and Manufacturing Engineering

Spain

rsanz@unizar.es

Cite this article: Sanz-Segura, R., Manchado Pérez, E., Özcan, E. (2019) 'Alarm Compliance in Healthcare: Design Considerations for Actionable Alarms (In Intensive Care Units)', in Proceedings of the 22nd International Conference on Engineering Design (ICED19), Delft, The Netherlands, 5-8 August 2019. DOI:10.1017/dsi.2019.88 


\section{INTRODUCTION}

Intensive care units are technologically advanced environments that are designed to safeguard the patient while their vitals are stabilized for further treatment. Audible and visual alarms are part of the healthcare ecology. Every electronic device (ranging from patient monitor to infusion pumps or bed heaters) has alarms incorporated that signal a technical or bodily malfunction. These alarms are part of the medical protocol designated by policy makers, international standards office, medical institutions, and manufacturers, and are rooted in complex system design that requires clinician action (Edworthy, 2013). However, audible alarms are so many that clinicians suffer from a syndrome called 'alarm fatigue' and often do not comply with the task alarm is conveying (Kristensen, Edworthy and Özcan, 2017).

Safe compliance behaviour is described as behaviour which do not violate policy, organization security rules and procedures such as taking security precautions and reporting any security incidents exist in an organization system (Humaidi and Balakrishnan, 2015). Measuring compliance with rules in the workspace and determining the success of a system belongs to the field of ergonomics and is based on data collected through task observations and scoring. Compliance is a term frequently used in medicine (as a synonymous of adherence or capacitance) to measure or explain the degree to which a patient correctly follows medical advice. At the same time, it also refers to the use of medical device or the response to healthcare procedures (De Geest and Sabaté, 2003). Compliance, when applied to critical medical alarms refers to timely acting on the message the alarm conveys. However, the phenomenon 'alarm fatigue' observed in intensive care units indicates that clinicians' non-compliance with alarms is a specific issue and can have serious life-threatening or long-term consequences on the patient safety and wellbeing.

In this paper, we will explore compliance with critical alarms by not only from their potential success or failure perspective (ergonomics measures and information theory) but also from the perspectives of the clinician capacity, needs, and motivations to comply with alarms in critical environments (e.g., behaviour change, persuasive model, appraisal theory). We will finally, reflect on further possible design strategies to increase compliance in critical care that are beyond following rules per se but through intrinsic motivation.

\section{WHAT IS COMPLIANCE IN HEALTHCARE}

While compliance in other industries can threaten mission safety and have financial risks, compliance in healthcare comes with even higher stakes: if clinicians fail to follow the proper procedure, they can end up injuring a patient or another staff member (PowerDMS, 2017). According to Manzey (2014), "compliance refers to the extent operators respond to a given alarm in accordance with the alarm, i.e. by initiating all actions necessary in case of a critical event or malfunction. Conversely, reliance refers to the extent to which operators refrain from any action unless the alarm goes off'. Compliance in medical care in hospitals can cover a wide variety of practices and be affected by various factors. In this particular context, nurses are the first to respond to the patient's needs, supervise the monitoring of their constants, responsible for keeping operations running smoothly and make sure that adequate procedures are followed and expectations are understood. Clinical alarms are one of the main channels to communicate a warning event to which nurses have the obligation to respond to, in order to guarantee patient safety (IEC 60601-1-8). At the same time, fatal incidents related to alarms are well documented in the literature. In the period 2005-2008, US Food and Drug Administration received 566 reports of alarm-related patient deaths (Joint Commission, 2013). Moreover, lack of compliance induces excessive number of alarms asking for attention eventually creating a chaotic environment. Patient sleep deprivation, stress and fatigue can be a potentially important factors in the pathogenesis of ICU delirium, which in turn is highly associated with increased patient mortality and morbidity. Thus, non-compliance can have both direct and indirect consequences threatening patient safety as well as well-being.

If medical alarms are designed to bring nurses to action, they can also be considered as stressors or stimuli that evoke an emotional episode for action. From Hancock's perspective on stress, alarm compliance can also be considered as a same interactive process with three facets (Hancock, 2008). First, the physiological internal response to a critical event, visible or invisible to the rest of individuals; second, the psychological evaluation or appraisal of the event, and the consequent 
response to task demand in function of mental workload, which may lead to care about the task or refuse it; and third, how the response is affected by a change in the environment. As it was identified by Hancock and Warm (1989), "the behaviour in response to a task is itself directly reflective of the stress level experienced" and consequently, the degree of (non)compliance for the mission performance. Clinicians experiencing alarm fatigue may also be suffering from lack of stimuli that bring them to action. The reasons for such non-action and the lack of compliance will be explain in the following paragraphs.

\section{WHY THERE IS NON-COMPLIANCE IN HEALTHCARE}

A significant number of accidents are caused by human errors due to the non-compliance with procedures such as ignoring important actions or getting confused by messages. The most common symptom of non-compliance in critical healthcare environments is the alarm fatigue. Here, we state that the term "non-compliance" means that clinicians fail to follow the directions due to conscious or unconscious behaviour or (in)voluntary ignorance of instructions, to the detriment of a beneficial experience for the organization or community (see also Kristensen, Edworthy, Özcan, 2017).

But, why is there non-compliance in the intensive care practitioners? There are several schools of studies (i.e., ergonomics, behavioural studies, alarm management) that give insights to explain why nurses often fail to follow the procedures. From these studies and in-situ observations it is derived that not all alarms that a nurse hears are medically actionable, which causes an inappropriate response to the warning event and induces disinterest and a lack of compliance. Intensive Care Units (ICUs) are a complex healthcare environment that involve multiple users, regulatory requirements, overlapping critical events and large amounts of data to be processed. Therefore, in such a critical context, audible alarms are the main medical alerts to warn clinicians of potential hazard events. The use of alarm sounds to prevent patients from dying or being seriously harmed is an integral part of modern care in hospitals. Alarms are fundamentally designed to be a crucial work tool for clinicians. Ironically, the excessive amount of all these alarms create a stressful sound environment. Clinicians hear multitude of alarms at the ICU, some of them come from their own patients, others from their colleagues. As a consequence, patients' mental and physical health as well as clinicians' compliance are threatened (Critical Alarms Lab, 2018). Other aspects as the location of the system unit layout or the floor plan also affects nurses' workload and explains why there is non-compliance in critical care.

Alarm compliance relate to alarm management by the organization and is measured by the lack of action on alarms. However, non-compliance cannot be attributed solely to ergonomic factors. There are also circumstantial issues and context-related attitudes and practices, caused by environment, social and organizational factors. Sowan (2015) concluded that "clinicians" attitudes and practices related to clinical alarms are key in designing contextually sensitive quality initiatives to fight alarm fatigue and therefore, an additional approach to consider in improving compliance. These factors, among others, are the level of stress tolerance, nurses experience level or training, the sensitivity to sound and the alarm nuisance degree, or the level of intuition (Humaidi, 2015; Park, 2003). Thus, compliance can be described from different approaches: from an ergonomic discipline, as adhering to requirements, standards, laws or regulations that relate to alarm management in order to guarantee the patient safety, based on human factors principles and previous expected values considerations (Meyers, 2004; Phansalkar, 2014); and/or from a persuasive model, to help and motivate people to adopt behaviour and/or change attitudes towards a more desirable outcome in critical care. The latter is more likely to be adopted by nurses than the mandatory approach, due to its more human-centred nature.

\subsection{Context-related factors inducing non-compliance}

According to Hancock $(2008,1989)$, further progress to predict someone's response in a specific stressful critical situation lies in two factors. The first one is related with a global issue about the complexity with interactions and its effects associated with workload and fatigue; the second one is the context-related issue where, due to the individual differences, a standardized response cannot be expected. This ecological approach (Gibson, 1979) is confirmed with intensive care practitioners and explains why non-compliance is happening. Thus, the personal appraisal processes of the available resources and the external events that affect tasks demanding can be summarized in the following 
specific factors observed in-situ in the daily life in intensive care units during the decision-making process.

\subsubsection{Patient-induced alarms}

Certain alarms are caused by a movement the patient make, or when patients are being treated and equipment is handled by nurses or doctors. Frequently patients induced an alarm asking for attention or simply, they sneeze or cough which triggers an unnecessary alarm. In such cases the alarm does not represent what is really happening to the patient.

\subsubsection{Sensor-induced technical alarms}

Sensors that are used to monitor patients may get disconnected or may malfunction. Examples are the oxygen saturation sensor on the patient's finger that is disconnected or sensors coming off during patient hygiene process and an alarm goes off. Though it is important to know that all sensors work, alarms also occur when nurses know the sensor has come off during treatment.

\subsubsection{Nurses' individual needs and preferences for alarm setting}

Nurses can influence the number of alarms they hear by changing the boundaries at which the medical device triggers an alarm for a certain value. However, nurses may set limits too narrow that results in a non-actionable alarm. An alarm that should usually be associated with a problem with for example one of the patient's vitals, can now actually mean a patient is responding as expected to medication. Each nurse has their own approach to these settings; that is, a personal preference in alarm management and several reasons for why they might choose one over the other. Thus, the alarms and the attention they ask of the nurse do not take into account how much information a nurse already has or need.

\subsubsection{Incomplete information from patient monitor}

The patient monitor signals an alarm based on a range of vitals that are measured. Since each of these values has a set of boundaries, the alarm will often go off when just one of these values exceeds the limit. The nurse however, needs more nuanced information about the combination of several values to be able to judge the urgency of the problem. The result is that most of the alarms the monitor produces are switched off because they do not require action.

\subsubsection{Cry-wolf effect}

Non-actionable alarms lead to desensitization to alarms, meaning that clinicians stop hearing the alarms or the alarms lose their meaning as clinicians repeatedly experience the alarms as false alarms. Cry-wolf effect may result in missed fatal alarms.

"Whether or not a stressor is experienced as discomforting is influenced by a variety of personal and contextual factors including capacities, skills and abilities, constraints, resources, and norms (Mechanic, 1978)". In order to improve alarm compliance by clinicians, it is necessary to study the variety of personal and contextual factors that affect their responses and actions in critical environments. Considering that strict protocols and traditional procedures fail to help clinicians to react at all or in a timely fashion. Because of this, alternative approaches are need to convince clinicians that the alarm is true and needs action.

\section{HOW TO IMPROVE COMPLIANCE IN CRITICAL CARE}

While non-compliance needs to be resolved in a multi-stakeholder setting beyond 'alarm management' with procedures and protocols, in this paper we focus on the 'alarm user' with their capacity and willingness to respond. A starting point to understand the human response in critical situations is the study of decision-making processes, available behavioural models and psychological theories that evaluate perception and emotional reaction for a specific event. Thus, a multidisciplinary inquiry must be considered in order to obtain design principles that guide alarm management in terms of compliance. Some of the theories to be considered as part of this multidisciplinary approach are shown in Table 1 and also detailed further. 
Table 1. Multidisciplinary approaches for alarm compliance analysis

\begin{tabular}{|l|c|r|}
\hline Model & \multicolumn{1}{|c|}{ Main content } & Some reference authors \\
\hline Persuasive models & $\begin{array}{c}\text { Affect user behaviours } \\
\text { or attitudes by means } \\
\text { of providing } \\
\text { knowledge }\end{array}$ & $\begin{array}{r}\text { (Orji, 2016) } \\
\text { (Nakajima, 2011) } \\
\text { (Oinas-Kukkonen, 2009) }\end{array}$ \\
\hline $\begin{array}{l}\text { Behavioural models } \\
\text { and theories of } \\
\text { change }\end{array}$ & $\begin{array}{c}\text { Affect user response } \\
\text { by means of specific } \\
\text { factors (motivation, } \\
\text { ability and triggers) }\end{array}$ & $\begin{array}{r}\text { (Fogg, 2002) } \\
\text { (Prochaska, 1997) }\end{array}$ \\
\hline Appraisal theory & $\begin{array}{c}\text { Affect user response } \\
\text { by means of } \\
\text { anticipating their } \\
\text { response under stress, } \\
\text { emotional influence } \\
\text { that can affect their } \\
\text { decision-making } \\
\text { processes }\end{array}$ \\
\hline $\begin{array}{l}\text { Communication } \\
\text { models }\end{array}$ & $\begin{array}{c}\text { Affect user response } \\
\text { by means of providing } \\
\text { proper and precise } \\
\text { understanding of the } \\
\text { message related to } \\
\text { each alarm }\end{array}$ & (Shannon and Weaver, 1949) \\
(Kellermann, 1992)
\end{tabular}

\subsection{Persuasive models}

Persuasive models pertaining to computing technology and interactive systems are a current and emerging trend for health promotion and prevention (Orji, 2016) but also for predicting user acceptance and to change user behaviours or attitudes (Oinas-Kukkonen, 2009). Studies in the fields of arts or psychology propose challenging and visionary approaches defining design principles and guidelines that support behaviour change through the user/operator's current behaviour or attitude (Fogg, 2002). Some of the best practices are based on providing users with feedback (their own behavioural mirror) and suitable support to achieve significant behaviour change regarding their choices and actions (Nakajima, 2011); improving interactivity of the information systems for a better communication (Matthews, 2015); or encouraging operators by an enhanced system through prompts, suggestions and reminders in order to motivate or engage users to particular behaviour (OinasKukkonen, 2009). In contrast with these approaches, auditory alarms carry too little information; in the absence of a meaningful message, nurses rely on a mental map of their patient and use this for their own interpretation and to prioritize alarm urgency on their own.

\subsection{Behavioural models and theories of change}

These are different attempts, and also complementary to each other, in order to understand specific behaviours by identifying the underlying factors and how response behaviours can change respectively. The transtheoretical model of behaviour change (TTM) is based on a temporal dimension, integrating processes and principles of change through stages, and from different interventions to change a user's undesirable behaviour (Prochaska, 1997). The Fogg Behaviour Model (FBM) devised for interaction design suggests behaviour as a result of three different factors: motivation, ability and triggers (Fogg, 2009). If the cognitive load is too high due to overlapping tasks, it is more difficult to focus on alarms and the ability to perform the task decreases. Triggers can be applied as reminders in clinical alarms exploring alternative modality channels. Undesired clinical behaviour can be solved understanding enables and barriers perceived by nurses. Prospective design projects as CareTunes by Koen Bogers or the Silent ICU by Elif Özcan, among others (see Critical alarms Lab projects) propose design opportunities that could lead to a behavioural change within the ICU. They are based on alternative solutions such as musical streaming monitoring or alarm 
communication removing sounds. They are different motivators to conduct more pleasant and quieter critical care environments that guarantee alarm compliance.

\subsection{Appraisal theory}

The appraisal theory is the evaluation, from the field of psychology, of the personal significance and meaning of an object or situation (Scherer, 1999), and the emotional reaction for a specific event. It describes the psychological steps of how 'stressors', -in this case "alarms"-, would possibly result in coping behaviour and reappraisal. As an extension of this theory, Richard Lazarus (1989) developed the transactional model of stress and coping. It describes how stimuli can be perceived by people. Depending on a person's appraisal of the stressor and their available resources to respond, they can experience stress. The model proposes consecutive stages that describe a decision making process. Perception filter stage is explained in a clinical environment with lots of stressors, where human filter is a survival mechanism. Nurses are extremely sensitive for alarms and filtering happens both consciously and unconsciously, being for some people harder to filter than for others. The primary reaction that a nurse has when an alarm sounds is to appraise the situation according to the criticality of the event and thus establish the level of danger. If an alarm is perceived as a false alarm and no attention is required, there is an opportunity to remove that alarm and become the stressor into a different design solution to communicate the event. Process-oriented models of appraisal theory are rooted in the idea that it is important to specify the cognitive principles and operations underlying these appraisal modes but also to define environmental factors that facilitate or inhibit action.

\subsection{Communication models}

A transactional model, in terms of communication, refers to a bidirectional interaction, from one person or system to another and back. In addition to the models mentioned previously, an understanding of what happens during the information processing is required, in order to improve the predisposition for alarm processing and to keep nurses in the loop. There is still a little known about the correlation between the information (system events or messages to communicate), the cues followed to guide medical design recommendations and the decisions reached by nurses in the context of clinical practice. In this sense, communication models are also useful to fragment the alarm communication process in different stages and anticipate the response of nurses and desired decisions in the latter stage (Shannon and Weaver, 1949; Kellermann, 1992). Thus, together with more recently available persuasive and behaviour models and theories of change, communication models can be taken as a starting point to define more successful actionable alarm design principles.

\section{CONCLUSIONS}

The aim of this contribution is to present a wider perspective in alarm management in healthcare scenarios, in order to explain clinicians' non-compliance behaviour. In order to address this, we present a multidisciplinary approach based not only in ergonomics, but also in the combination of studies about decision-making processes, existing persuasive and behavioural models and psychological theories. These alternative approaches help clinicians to adopt a more desirable behaviour and motivating attitudes with critical alarms.

This paper focuses on the 'alarm user' with a human-centred approach in order to provide knowledge for designers and all the stakeholders involved in alarm compliance (device manufactures, medical institutions and clinicians, or policy makers). From this study, those disciplines can obtain new insights into designing better alarms, understanding the work environment in which clinicians in general, and intensive care practitioners in particular, operate in order to: reorganise the floorplan layout, manage the information system, or to develop new design strategies that increase compliance, not based only on the mandatory nature but also in human-centred approaches with motivation as an underlying challenge. From current knowledge and in-situ observations in several critical care areas, it highlights the need to be aware of the emotions and behaviour responses in a deeper way and from an individual diverse appraisal, considering the human factors that make ICU a complex scenario.

The literature collected provides insights into future innovative solutions to take as reference in order to increase compliance in such a positive way that clinicians want to respond to alarms. The persuasive approach gathers design principles useful in a clinical alarm context such as an emotional 
and meaningful impact in engaging in behavioural change (e.g., the use of alternative sensory or cognitive pathways, such as musical or artistic languages, can be the solution to restore a peaceful environment for patients to recover in an intensive care unit). Alternative modalities of user interaction, from passive monitoring behaviour through smart devices to a slow technology philosophy implement user-sensitive systems that can sense the people present in a patient room, adapting their communication systems to the different needs of who use them. Reducing pre-emptive alarms using artificial intelligence for filtering true alarms; allowing a system for nurses to become proactive, rather than reactive; or the need of keep nurses into a feedback loop are other positive trends to consider for alarm compliance.

Traditional research in literature in processes related to decision-making suggest a linear processing that is simple and autonomous, whereas the reality in medical practice is about organizational relationships and complex processes that require a full circle of actions with emotional, contextual and personal characteristics affecting task performance. However, healthcare and ICUs are not the only critical area that requires an improvement in compliance with alarms. Emergency situations, mission control rooms, aircrafts cockpits or autonomous driving are dynamic systems and all require very often time-critical responses (Sousa et al., 2016; Baldwin \& Lewis, 2014; Heydra, Jansen \& Van Egmond, 2014; Graham, 1999; Sorkin, 1988; Patterson, 1982). The aim of any alarm design framework to fight non-compliance in critical contexts, should be to provide the best evidence about the management of a critical event informing operators (nurses), and e.g. patients where possible, about options that are available, together with their risks and benefits (Hancock, 2005). The response of a nurse is highly context-related; a critical environment governed by multitude of auditory (and visual) warnings, not prioritized, difficult to distinguish and that cause the recurring alarm fatigue.

As an alternative to traditional linear models, we are currently developing a circular framework for nurse-centred compliance towards audible alarms. This contribution is one of the starting points to enrich the entire ecology of the alarm communication process in critical care. Future directions are aimed at filling the gap in the last stage of the communication process and give designers the tools to design new strategies during the alarm design process, from a nurse-centred approach and in the search for a more desirable outcome in critical care. Thus, we will feel successful when we close loop by motivating nurses to comply with an audible alarm that is reliable, context-relevant, and is seamlessly embedded in the complex technological architecture of intensive care units.

\section{ACKNOWLEDGEMENTS}

We would like to thank the hardworking clinicians at Erasmus Medical Centre Rotterdam (NL), Hospital Quirón Zaragoza and Hospital HLA Montpellier Zaragoza (SP) for providing us with opportunities for discussions and interviews on the topic of alarm fatigue. We also thank the researchers at Critical Alarms Lab (TU Delft, NL) for their inspirational work on designing against alarm fatigue.

\section{REFERENCES}

Baldwin, C. L. and Lewis, B. A. (2014), "Perceived urgency mapping across modalities within a driving context", Applied Ergonomics, Vol. 45 No. 5, pp. 1270-1277. https://doi.org/10.1016/j.apergo.2013.05.002

De Geest, S. and Sabaté, E. (2003), “Adherence to long-term therapies: Evidence for action”, European Journal of Cardiovascular Nursing, Vol. 2 No. 4, pp. 323-323. http://dx.doi.org/10.1016/s1474-5151(03)00091-4.

Edworthy J. (2013), J Am Med Inform Assoc, Vol. 20, pp. 584-589. http://doi.org/10.1136/amiajnl-2012-001061

Fogg, B. J. (2002), "Persuasive technology: using computers to change what we think and do", Ubiquity, Vol. 5. https://doi.org/10.1145/764008.763957

Fogg, B. J. (2009), "Creating persuasive technologies", Proceedings of the 4th International Conference on Persuasive Technology - Persuasive'09. http://dx.doi.org/10.1145/1541948.1542005.

Gibson, J. (1979), The Ecological Approach to Human Perception. https://doi.org/10.1002/bs.3830260313

Graham, R. (1999), "Use of auditory icons as emergency warnings: evaluation within a vehicle collision avoidance application", Ergonomics, Vol. 42 No. 9, pp. 1233-1248.

Hancock, H. C. and Easen, P. R. (2006), "The decision-making processes of nurses when extubating patients following cardiac surgery: an ethnographic study", International Journal of Nursing Studies, Vol. 43 No. 6, pp. 693-705. https://doi.org/10.1016/j.ijnurstu.2005.09.003

Hancock, P. A. and Szalma, J. L. (Eds.) (2008), Performance under Stress. Ashgate Publishing, Ltd. 
Hancock, P. A. and Warm, J.S. (1989), “A dynamic model of stress and sustained attention”, Human Factors, Vol. 31 No. 5, pp. 519-537. https://doi.org/10.1177/001872088903100503

Heydra, C. G., Jansen, R. J. and Van Egmond, R. (2014), “Auditory signal design for automatic number plate recognition system", In Creating the Difference: Proceedings of the Chi Sparks 2014 Conference, The Hague The Netherlands, 13 April 2014. The Hague University of Applied Sciences.

Humaidi, N. and Balakrishnan, V. (2015), "Leadership styles and information security compliance behavior: The mediator effect of information security awareness", International Journal of Information and Education Technology, Vol. 5 No. 4, p. 311. https://doi.org/10.7763/ijiet.2015.v5.522

Kellermann, K. (1992), "Communication: Inherently strategic and primarily automatic”, Communications Monographs, Vol. 59 No. 3, pp. 288-300. https://doi.org/10.1080/03637759209376270

Kristensen, M., Edworthy, J. and Özcan, E. (2017). "In search of acoustical signs of alarm fatigue", Sound Effects, pp. 88-104.

Joint Commission. (2013), "Medical device alarm safety in hospitals", Sentinel Event Alert, No. 50, p. 1.

Lazarus, R. S. (1990), “Theory-based stress measurement”, Psychological Inquiry, Vol. 1 No. 1, pp. 3-13. https://doi.org/10.1207/s15327965pli0101_1

Manzey, D., Gérard, N. and Wiczorek, R. (2014), "Decision-making and response strategies in interaction with alarms: the impact of alarm reliability, availability of alarm validity information and workload", Ergonomics, Vol. 57 No. 12, pp. 1833-1855. https://doi.org/10.1080/00140139.2014.957732

Matthews, J. et al. (2016), "Persuasive technology in mobile applications promoting physical activity: a systematic review", Journal of Medical Systems, Vol. 40 No. 3. http://dx.doi.org/10.1007/s10916-0150425-x.

Mechanic, D. (1978), "Effects of psychological distress on perceptions of physical health and use of medical and psychiatric facilities", Journal of Human Stress, Vol. 4 No. 4, pp. 26-32. https://doi.org/10.1080/0097840x.1978.10545983

Nakajima, T. and Lehdonvirta, V. (2011), "Designing motivation using persuasive ambient mirrors", Personal and Ubiquitous Computing, Vol. 17 No. 1, pp.107-126. http://dx.doi.org/10.1007/s00779-011-0469-y.

International Electrotechnical Commission. Medical electrical equipment, part 1-8: General requirements, tests and guidance for alarm systems in medical electrical equipment and medical electrical systems. 2005, Geneva. IEC 60601-1-8. International Electrotechnical Commission, Geneva, 2006.

Oinas-Kukkonen, H. (2009), "Persuasive systems design: key issues, process model, and system features", Communications of the Association for Information Systems, Vol. 24. http://dx.doi.org/10.17705/1cais.02428.

Orji, R. (2016), Preface to the International Workshop on Personalization in Persuasive Technology: Research Challenges and Opportunities Strategies. In PPT@ PERSUASIVE (pp. 1-5).

Özcan, E. (2018), Critical Alarms Lab. [online] Delft University of Technology. Available at: (https://delftdesignlabs.org/criticalalarmslab/) (13th December 2018).

Patterson, R. D. (1982), "Guidelines for auditory warning systems on civil aircraft”, Civil Aviation Authority.

Phansalkar, S., Zachariah, M., Seidling, H. M., Mendes, C., Volk, L. and Bates, D. W. (2014), "Evaluation of medication alerts in electronic health records for compliance with human factors principles", Journal of the American Medical Informatics Association, Vol. 21 No. e2, pp. e332-e340. http://dx.doi.org/10.1136/amiajnl-2013-002279.

PowerDMS. (December, 2017), Definition of Compliance in Healthcare. [online]. Available at: (https://www.powerdms.com/blog/definition-compliance-healthcare/) (13th December 2018).

Prochaska, J. O. and Velicer, W. F. (1997), "The transtheoretical model of health behavior change", American Journal of Health Promotion, Vol. 12 No. 1, pp. 38-48. http://dx.doi.org/10.4278/0890-1171-12.1.38.

Scherer, K. R. (1999), Appraisal Theory. Handbook of cognition and emotion, pp. 637-663. https://doi.org/10.1002/0470013494.ch30

Shannon, C. E. and Weaver, W. (1949), The Mathematical Theory of Communication, University of Illinois Press, Urbana, IL.

Sorkin, R. D. (1988), "Why are people turning off our alarms?", The Journal of the Acoustical Society of America, Vol. 84 No. 3, pp. 1107-1108. https://doi.org/10.1121/1.397232

Teixeira De Sousa, B., Donati, A., Özcan, E., van Egmond, R., Edworthy, J., Jansen, R. ... and Voumard, Y. (2016), "Designing and deploying meaningful audio alarms for control systems". In 14th International Conference on Space Operations (p. 2616). https://doi.org/10.2514/6.2016-2616

Sowan, A. K., Tarriela, A. F., Gomez, T. M., Reed, C. C. and Rapp, K. M. (2015), “Nurses' perceptions and practices toward clinical alarms in a transplant cardiac intensive care unit: Exploring key issues leading to alarm fatigue", JMIR Human Factors, Vol. 2 No. 1. https://doi.org/10.2196/humanfactors.4196 